\title{
EDUCAÇÃO EM SAÚDE
}

\section{E O APRIMORAMENTO DE}

NOVOS CONHECIMENTOS

\section{Organizador:}

Pablo Augusto Gurgel de Sousa

VOLUME 1

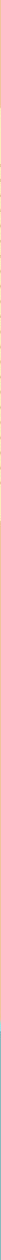




\section{EDUCAÇÃO EM SAÚDE}

\section{E O APRIMORAMENTO DE NOVOS CONHECIMENTOS}

Organizador:

Pablo Augusto Gurgel de Sousa

\section{VOLUME 1}

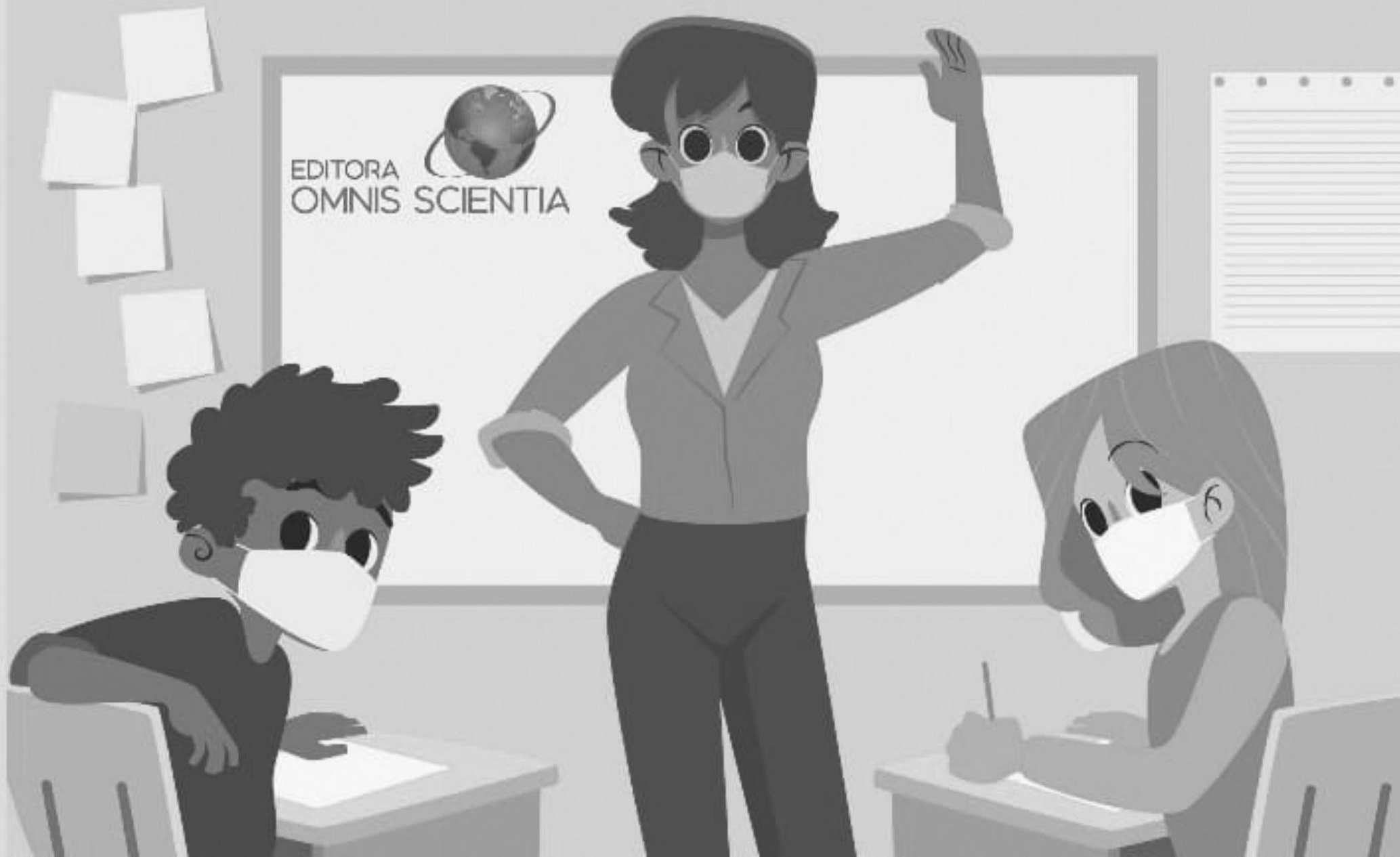


Editora Omnis Scientia

EDUCAÇÃO EM SAÚDE E O APRIMORAMENTO DE NOVOS CONHECIMENTOS

Volume 1

$1^{a}$ Edição

TRIUNFO - PE 


\section{Editor-Chefe}

Me. Daniel Luís Viana Cruz

Organizador

Me Pablo Augusto Gurgel de Sousa (Mestre em Psicobiologia)

\section{Conselho Editorial}

Dr. Cássio Brancaleone

Dr. Marcelo Luiz Bezerra da Silva

Dra. Pauliana Valéria Machado Galvão

Dr. Plínio Pereira Gomes Júnior

Dr. Walter Santos Evangelista Júnior

Dr. Wendel José Teles Pontes

Editores de Área - Ciências da Saúde

Dra. Camyla Rocha de Carvalho Guedine

Dra. Cristieli Sérgio de Menezes Oliveira

Dr. Leandro dos Santos

Dr. Hugo Barbosa do Nascimento

Dr. Marcio Luiz Lima Taga

Dra. Pauliana Valéria Machado Galvão

\section{Assistente Editorial}

Thialla Larangeira Amorim

Imagem de Capa

Freepik

\section{Edição de Arte}

Vileide Vitória Larangeira Amorim

\section{Revisão}

Os autores

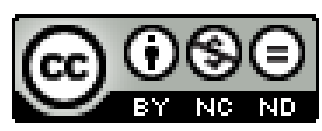

Este trabalho está licenciado com uma Licença Creative Commons - AtribuiçãoNãoComercial-SemDerivações 4.0 Internacional.

O conteúdo abordado nos artigos, seus dados em sua forma, correção e confiabilidade são de responsabilidade exclusiva dos autores. 


\title{
Dados Internacionais de Catalogação na Publicação (CIP)
} (eDOC BRASIL, Belo Horizonte/MG)

E24 Educação em saúde e o aprimoramento de novos conhecimentos [livro eletrônico] / Organizador Pablo Augusto Gurgel de Sousa. Triunfo, PE: Omnis Scientia, 2021.

145 p. : il.

Formato: PDF

Requisitos de sistema: Adobe Acrobat Reader

Modo de acesso: World Wide Web

Inclui bibliografia

ISBN 978-65-88958-61-2

DOI 10.47094/978-65-88958-61-2

1. Educação sanitária. 2. Saúde pública. 3. Qualidade de vida.

I. Sousa, Pablo Augusto Gurgel de.

CDD 362.1

Elaborado por Maurício Amormino Júnior - CRB6/2422

\author{
Editora Omnis Scientia \\ Triunfo - Pernambuco - Brasil \\ Telefone: +55 (87) 99656-3565 \\ editoraomnisscientia.com.br \\ contato@editoraomnisscientia.com.br
}




\section{PREFÁCIO}

Saudações prezado (a) leitor (a),

Em seu livro Dez Bilhões, publicado em 2013, o professor Stephen Emmott, de Oxford, indagou que "há 10.000 anos éramos apenas um milhão. Em 1800, faz pouco mais de 200 anos, já éramos um bilhão. Há 50, por volta de 1960, chegamos a 3,5 bilhões. Atualmente, superamos 7,5 bilhões”. Mais precisamente, segundo dados do novo relatório do Population Reference Bureau (PRB), somos cerca de 7,8 bilhões de seres humanos habitando este planeta no momento. Não obstante à visão apocalíptica deste panorama, a que se deve tamanha progressão geométrica?

Consenso entre historiadores e estudiosos da demografia humana, muito mais do que abandonar o modo de vida nômade, as descobertas e avanços da área médica foram fundamentais para que os séculos XX e XXI registrassem um elevado crescimento populacional. É notável que, a partir desse período, se consolidou e se difundiu a importância da pesquisa em saúde, não só com o objetivo de sanar doenças, mas também de prevenção e promoção à saúde, provendo ao indivíduo e à sociedade meios para a melhoria da qualidade de vida.

Nesta perspectiva, sabendo que o conhecimento científico é muito valioso, principalmente em um cenário pandêmico causado pelo vírus Sars-CoV-2, a Editora Omnis Scientia nos abrilhanta com o livro Educação em Saúde e o aprimoramento de novos conhecimentos. Por meio de um compilado de artigos, este constructo evidencia a importância do papel dos profissionais de saúde como divulgadores científicos, seja em pesquisas teóricas, aplicadas, de inovação tecnológica ou mesmo relatos de experiências, combatendo a cultura da desinformação, auxiliando a promoção de políticas públicas efetivas e refletindo sobre as nossas ações perante a sociedade como um todo.

Ademais, esta publicação surge em circunstância significativa como forma de promover o avanço, ainda mais expressivo, do processo de inserção do Brasil no patamar dos grandes centros científicos do mundo. Essa iniciativa, portanto, deve ser celebrada, além do mais, pela disseminação do conhecimento científico em educação em saúde, adequado em qualidade e momento oportuno, primordial para promoção do bem-estar populacional.

Por fim, em nossos livros, selecionamos um dos capítulos para premiação como forma de incentivo para os autores, e entre os excelentes trabalhos selecionados para compor este livro, o premiado foi o capítulo 2, intitulado "Residência multiprofissional em Saúde da Família e as contribuições para a interprofissionalidade e a formação do Assistente Social". Por meio de relato de experiência vivenciada pela residente de serviço social, o trabalho nos traz reflexões sobre as contribuições do programa de residência no processo de aprendizagem e qualificação profissional, bem como, para as ações multiprofissionais de educação em saúde, desenvolvidas em conjunto com os residentes de diversas áreas da saúde. 


\section{SUMÁRIO}

CAPÍTULO 1 .14

ENFRENTAMENTO À COVID-19 PELA EQUIPE MULTIPROFISSIONAL NA ATENÇÃO BÁSICA E SAÚDE DA FAMÍLIA

Patrícia Fernanda Faccio

Ântony Eliel Andrade da Silva

Brenda Fernanda Guedes

José Filipe da Silva

Kristine Kelly de Albuquerque

Maria Daniele da Silva

Marianne de Araújo Mendes

Mércia Fernanda Melo da Silva

Taise Maria da Costa

João Paulo Maciel Cavalcanti de Albuquerque

DOI: 10.47094/978-65-88958-61-2/14-20

CAPÍTULO 2

RESIDÊNCIA MULTIPROFISSIONAL EM SAÚDE DA FAMÍLIA: INTERPROFISSIONALIDADE E A FORMAÇÃO DO ASSISTENTE SOCIAL

Rafaela Zulmira de Oliveira Moraes

Christiani Cassoli Bortoloto Lopes

Andréia Santina Seubert Dalferth

Évelyn Farias

Estefany Bahert

Pedro Henrique de Carli

Maria Nazaré Murilho

Isabela Cristina Mannes

Danieli Cristina Scalco 
Felipe Gustavo de Bastiani

Yasmin Luisa Dengo Lombardo

Gisielli Jovenilia Polidorio Alievi

DOI: 10.47094/978-65-88958-61-2/21-37

CAPÍTULO 3. .38

AÇÃO EDUCATIVA COMO FERRAMENTA PARA PREVENÇÃO DO CÂNCER DE MAMA EM UMA UNIDADE DE ATENÇÃO PRIMÁRIA À SAÚDE

Matheus Gomes Andrade

Dilene Fontinele Catunda Melo

Maria Larysse Muniz Pereira

Lurdiane Gabriel Pereira

Maria Aparecida Melo Morais

Glória Vanessa de Araújo Silva Sousa

Jesus Carlos Eduardo de Paiva Avelino

Fernanda Alália Braz de Sousa

Maria das Graças Teodosio Dias

Viceni Almeida Ludgero

Rosângela Souza Cavalcante

Francisca Nellie de Paula Melo

DOI: 10.47094/978-65-88958-61-2/38-44

CAPÍTULO 4 .45

TRATAMENTO INTRALESIONAL DE LEISHMANIOSE CUTÂNEA LOCALIZADA (LCL) EM UNIDADE BÁSICA DE SAÚDE DURANTE PANDEMIA

Sarah Ramany Faria Salmeron

Daliany Santos

Adrielly Sousa Guimarães

Lucas Salvador Pereira 
CAPÍTULO 5. .51

ESTRATÉGIAS DE EDUCAÇÃO EM SAÚdE PARA CRIANÇAS EM TEMPOS DE PANDEMIA: RELATO DE EXPERIÊNCIA

Mirelly Shatilla Misquita Tavares

Clara de Sousa Rodrigues

Anna Beatriz de Almeida Gomes Sousa

Mikaelly Teixeira Alves

Naylton Morais Dias

Dannilo Dias Soares

Viceni Almeida Ludgero

Wagner da Costa Bezzerra

Fernanda Alália Braz de Sousa

Carlos Alberto Cavalcante de Lima

Mariane Pereira da Luz Melo

Samara Lais Silva Ferreira

Francisca de Fatima dos Santos Freire

DOI: 10.47094/978-65-88958-61-2/51-61

CAPÍTULO 6 .62

ATUAÇÃODAENFERMEIRANAEQUIPE MULTIPROFISSIONALNOTRATAMENTO DE CÂNCER: RELATO DE EXPERIÊNCIA

Carla Walburga da Silva Braga

DOI: 10.47094/978-65-88958-61-2/62-69 
EDUCAÇÃO EM SAÚdE BUCAL COMO ESTRATÉGIA DE PREVENÇÃO À CÁRIE DENTÁRIA NA INFÂNCIA

Lara Pepita de Souza Oliveira

Jardel dos Santos Silva

Jefter Haad Ruiz da Silva

Esaú Lucas Nascimento Tavares

Ivana Caroline de Souza Marinho Araújo

Luca Ramon da Silva Lima

Ivete Castro de Souza

Kerolayne Sena de Sousa Santos

Erika Akiko Moura Shiota

Dina Birman

Cristiane Maria Brasil Leal

Diego Ferreira Regalado

DOI: 10.47094/978-65-88958-61-2/70-79

CAPÍTULO 8. 80

\section{TÓPICOS RELEVANTES RELACIONADOS À SAÚDE DA MULHER}

Lília Barroso Cipriano de Oliveira

Rebeca Barroso Cipriano de Oliveira

Regizeuda Ponte Aguiar

DOI: 10.47094/978-65-88958-61-2/80-86

CAPÍTULO 9 .87

ESTRATÉGIA PARA EDUCAÇÃO EM SAÚDE EM TEMPOS DE PANDEMIA: RODA DE CONVERSA VIRTUAL SOBRE APLV

Ludmylla Rolim de Albuquerque

Mayara Vieira Rodrigues 
Bruna Ramalho Nogueira Diniz

Maria Luíza Formiga Barros Batista

Taynara Souza Santos

Núbia Kelly Rodrigues Ribeiro

Ideltônio José Feitosa Barbosa

DOI: 10.47094/978-65-88958-61-2/87-94

CAPÍTULO 10 .95

CONHECIMENTO DOS UNIVERSITÁRIOS DO SEXO MASCULINO SOBRE O USO DO ANTICONCEPCIONAL DE EMERGÊNCIA

Laryssa Bezerra Silva

Nathália Lima de Pontes

Graziani Izidoro Ferreira

Fernanda Souza e Silva Garcia

DOI: 10.47094/978-65-88958-61-2/95-101

CAPÍTULO 11

SAÚdE E TECNOLOGIA: A PERCEPÇÃO DE JOVENS RURAIS ACERCA DA TELESAÚDE NO CUIDADO EM PSICOTERAPIA

Isadora Ribas Strojarki

Marcelo Moreira César

Thalia Brites Muniz

Ana Carolina Ferraz

Dawid Da Silva Vargas

DOI: 10.47094/978-65-88958-61-2/102-116 
TELERREABILITAÇÃO COMO RECURSO FISIOTERAPÊUTICO NAATENÇÃO BÁSICA

FRENTE À PANDEMIA DE COVID - 19

Patrícia Fernanda Faccio

Alex Lira do Nascimento e Silva

Elaine Ferreira Silva

Samuel Cézar Alexandre Silva

Mércia Fernanda Melo da Silva

Giuliane Diógenes Norberto da Silva

Jullia Carolyne Rosa Cordeiro de Lima

Tatianny dos Santos Cassiano

Paula Drielly de Melo Ribeiro

Soraya Santos Alves Barbosa

João Paulo Maciel Cavalcanti de Albuquerque

DOI: 10.47094/978-65-88958-61-2 /117-125

CAPÍTULO 13.

USO DO INSTAGRAM COMO FERRAMENTA DE EDUCAÇÃO EM SAÚDE SOBRE APLV DURANTE A PANDEMIA DO COVID-19

Emanuel de Oliveira Colombo

Laysa Bianca Gomes de Lima

Abiel Reyfe da Silva Canuto

Núbia Kelly Rodrigues Ribeiro

Ideltônio José Feitosa Barbosa

DOI: 10.47094/978-65-88958-61-2/126-133 
CAPÍTULO 14.

VIABILIDADE DOS APLICATIVOS m-HEALTH PARA PACIENTES COM DOENÇAS PULMONARES CRÔNICAS NO SISTEMA PÚBLICO DE SAÚDE DO DISTRITO FEDERAL

Heloisa Glass

Gabriel Cordeiro Schimidt

Igor Louza Pereira

Paulo Henrique de Ramos Feitosa

DOI: 10.47094/978-65-88958-61-2 /134-141 


\section{CAPÍTULO 1}

\section{ENFRENTAMENTO À COVID-19 PELA EQUIPE MULTIPROFISSIONAL NA ATENÇÃO BÁSICA E SAÚDE DA FAMÍLIA}

\section{Patrícia Fernanda Faccio';}

Centro Universitário Tabosa de Almeida (ASCES - UNITA), Caruaru, Pernambuco. http://lattes.cnpq.br/2762370293220025

Ântony Eliel Andrade da Silva²;

Centro Universitário Tabosa de Almeida (ASCES - UNITA), Caruaru, Pernambuco. $\underline{\text { http://lattes.cnpq.br/2316891471710695 }}$

\section{Brenda Fernanda Guedes ${ }^{3}$;}

Centro Universitário Tabosa de Almeida (ASCES - UNITA), Caruaru, Pernambuco. $\underline{\text { http://lattes.cnpq.br/5887230214002859 }}$

\section{José Filipe da Silva ${ }^{4}$;}

Centro Universitário Tabosa de Almeida (ASCES - UNITA), Caruaru, Pernambuco. $\underline{\text { http://lattes.cnpq.br/2656332021326911 }}$

\section{Kristine Kelly de Albuquerque ${ }^{5}$;}

Centro Universitário Tabosa de Almeida (ASCES - UNITA), Caruaru, Pernambuco.

http://lattes.cnpq.br/9389300631803474

\section{Maria Daniele da Silva ${ }^{6}$;}

Centro Universitário Tabosa de Almeida (ASCES - UNITA), Caruaru, Pernambuco. http://lattes.cnpq.br/0497272541563920

\section{Marianne de Araújo Mendes ${ }^{7}$;}

Centro Universitário Tabosa de Almeida (ASCES - UNITA), Caruaru, Pernambuco. http://lattes.cnpq.br/0835960171862297

\section{Mércia Fernanda Melo da Silva}

Centro Universitário Tabosa de Almeida (ASCES - UNITA), Caruaru, Pernambuco. http://lattes.cnpq.br/2027202592844623 


\section{Taise Maria da Costa";}

Centro Universitário Tabosa de Almeida (ASCES - UNITA), Caruaru, Pernambuco. $\underline{\text { http://lattes.cnpq.br/5584519395547998 }}$

\section{João Paulo Maciel Cavalcanti de Albuquerque ${ }^{10}$.}

Centro Universitário Tabosa de Almeida (ASCES - UNITA), Caruaru, Pernambuco. http://lattes.cnpq.br/2716963807209565

RESUMO: A COVID-19 se apresentou com rápida disseminação devido suas características de transmissão por contato com aerossóis contaminados, produzindo uma curva epidêmica alta em um curto espaço de tempo, sobrecarregando sistemas de saúde no Brasil e no mundo. O Sistema Único de Saúde (SUS) é universal, gratuito, regionalizado e de ampla cobertura, sendo que aproximadamente $80 \%$ da população brasileira depende exclusivamente dele para acessar ações e serviços de saúde. A integralidade é um de seus princípios, assegurando ações de promoção, prevenção, diagnóstico, tratamento e reabilitação, integradas por meio de uma rede. Com a notável importância da rede hospitalar, o SUS foi colocado em evidência durante a pandemia, mas é preciso fortalecer a importância da Atenção Básica e suas possibilidades de cuidado, sendo está a linha de frente para orientação populacional e comunitária e diagnóstico precoce à COVID-19. Este estudo objetiva relatar experiências da Atenção Básica no enfrentamento à COVID-19 vivenciada pela Equipe Multiprofissional em Saúde da Família no Município de Caruaru - PE. A metodologia consiste em relato de experiência das estratégias aplicadas para continuidade do serviço, alinhadas com o cumprimento das recomendações sanitárias. As estratégias foram: (1) Planejamento e reuniões remotas com a equipe multiprofissional para criação de possibilidades de continuidade ao cuidado (2) Divisão dos horários de atuação da equipe para não sobrecarregar os espaços (3) Identificação de usuários mais vulneráveis de acordo com suas necessidades de saúde (4) Solicitação para uso de máscara e álcool em gel pelos usuários na unidade e em suas casas (5) Teleconsulta e Telerreabilitação de forma síncrona. As intervenções aplicadas possibilitaram a manutenção do cuidado com a população durante a pandemia. A Residência Multiprofissional em Saúde da Família exemplifica o potencial que a Atenção Básica desempenha, unindo ensino e serviço para fortalecimento da gestão em saúde e do enfrentamento da pandemia da COVID-19.

PALAVRAS-CHAVE: Atenção Primária à Saúde. Sistemas de Saúde. COVID-19. 
ABSTRACT: COVID-19 showed rapid dissemination due to its transmission characteristics through contact with contaminated aerosols, producing a high epidemic curve in a short period of time, overloading health systems in Brazil and in the world. The Sistema Único de Saúde (SUS) is universal, free, regionalized and with wide coverage, and approximately $80 \%$ of the Brazilian population depends exclusively on it to access health actions and services. Integrality is one of its principles, ensuring promotion, prevention, diagnosis, treatment and rehabilitation actions, integrated through a network. With the remarkable importance of the hospital network, the SUS was highlighted during the pandemic, but it is necessary to strengthen the importance of Primary Care and its possibilities of care, as this is the front line for population and community guidance and early diagnosis to COVID19. This study aims to report experiences of Primary Care in coping with COVID-19 experienced by the Multiprofessional Team in Family Health in the city of Caruaru - PE. The methodology consists of an experience report of the strategies applied for the continuity of the service, in line with the fulfillment of health recommendations. The strategies were: (1) Planning and remote meetings with the multidisciplinary team to create possibilities for continuity of care (2) Division of the team's working hours so as not to overload the spaces (3) Identification of the most vulnerable users according to their health needs (4) Request for the use of mask and alcohol gel by users in the unit and in their homes (5) Synchronous Teleconsultation and Telerehabilitation. The interventions applied made it possible to maintain care for the population during the pandemic. The Multiprofessional Residency in Family Health exemplifies the potential that Primary Care plays, uniting teaching and service to strengthen health management and fight the COVID-19 pandemic.

KEY-WORDS: Primary Care. Health Systems. COVID-19.

\section{INTRODUÇÃO}

A COVID-19 é uma doença infecciosa causada pelo coronavírus SARS-CoV-2, apresentando quadro clínico que pode variar entre sintomas leves, moderados e graves, ou ainda em quadros assintomáticos. O vírus se propaga por meio de contato direto com aerossóis, sendo por meio de superfícies e objetos ou proximidade com pessoas contaminadas. O tempo entre a exposição e o surgimento de sintomas transcorre entre 2 e 14 dias, período que é denominado de incubação (BRASIL, 2020b).

Devido a tais características, a COVID-19 se apresentou com rápida disseminação, produzindo uma curva epidêmica alta em um curto espaço de tempo, sobrecarregando sistemas de saúde no Brasil e no mundo. Com a notável importância da rede hospitalar, o SUS foi colocado em evidência durante a pandemia, mas é preciso fortalecer a importância da atenção básica e suas possibilidades de cuidado, sendo esta a linha de frente para orientação populacional e comunitária e diagnóstico 
precoce à COVID-19. O monitoramento de casos leves em isolamento domiciliar e a identificação precoce de sinais de alerta são ferramentas de controle da propagação e letalidade da doença que podem ser articulados na atenção básica (CAMPOS, et al., 2020).

O Sistema Único de Saúde (SUS) é universal, gratuito, regionalizado e de ampla cobertura, sendo que aproximadamente $80 \%$ da população brasileira depende exclusivamente dele para acessar ações e serviços de saúde. A integralidade é um de seus princípios, assegurando ações de promoção, prevenção, diagnóstico, tratamento e reabilitação, integradas por meio de uma rede. Busca-se solucionar os problemas da população a partir da realidade local do território e da população adscrita, além de fortalecer vínculos sociais, comunitário, familiares e intersetoriais. Como porta de entrada da rede de saúde, norteia-se pela continuidade e responsabilização do cuidado, com ações humanizadas, equânimes e de participação social (BRASIL, 2012).

Campos et al. (2020) apontam que o fortalecimento da atenção básica através de qualificação de recursos humanos e organização da rede contribuem diretamente na redução de morbimortalidade das populações adscritas, consequentemente impactando nos índices de saúde a nível macro. Dessa forma, o presente estudo se justifica pela notável importância e necessidade de propagar orientações com base científica a profissionais e usuários/as do SUS sobre as medidas de prevenção e enfrentamento à COVID-19, assim como contribuir para constante atualização e aprimoramento de práticas de produção de saúde e compartilhamento de experiências efetivas na realidade brasileira.

\section{METODOLOGIA}

O estudo se guia por abordagem qualitativa de pesquisa, através de natureza básica e método descritivo de relato de experiência aliado à pesquisa-ação. Entende-se por pesquisa-ação o método de pesquisa social com base empírica, no qual o/a(s) pesquisador/a(s) parte(m) de uma realidade concreta com a finalidade de atuar ativa e diretamente na direção de solucionar uma questão específica. Ainda, tal investigação-ação é atualizada de forma contínua, sistemática e empiricamente fundamentada, buscando assim aprimorar teoria e prática de forma interdependente (TRIP, 2005).

O local do estudo foram os territórios de atuação da equipe multiprofissional do programa de residência em atenção básica e saúde da família, vinculado ao Centro Universitário Tabosa de Almeida (ASCES - UNITA), no município de Caruaru-PE. O período de atuação do referido relato corresponde aos meses de março, abril e maio do presente ano.

\section{RESULTADOS E DISCUSSÕES}

Estratégia 1. Ações de planejamento são necessárias para diagnosticar a realidade e propor alternativas para transformá-la, assim como pensar nos meios para que isso se viabilize. Assim sendo, pensando na coletividade das áreas da saúde e das populações por elas atendidas, o planejamento se torna essencial para gerenciar demandas, expectativas e estratégias a fim de melhorar os índices de saúde do território. O planejamento e a realização de reuniões remotas com a equipe multiprofissional 
para criação de possibilidades de continuidade ao cuidado atenderam tal premissa a nível micro e local, buscando integrar a resolutividade e a corresponsabilidade de forma ética e com visão ampliada, conforme apontam Junges, Barbiani e Zoboli (2015).

Estratégia 2. A divisão dos horários de atuação da equipe multiprofissional para não sobrecarregar os espaços das unidades de saúde corrobora com as medidas sanitárias preventivas de distanciamento social. O distanciamento social pela população, aliado ao isolamento social das pessoas contaminadas, é a medida mais efetiva de proteção social e deve ser fortalecida conjuntamente pela atenção básica e pelas ações de vigilância em saúde, visando assim reduzir a propagação e transmissão do vírus (AQUINO, et al., 2020).

Estratégia 3. A identificação de grupos vulneráveis e prioritários de acordo com suas necessidades de saúde, bem como o acompanhamento de condições crônicas, são ações de fundamental importância para controle da morbimortalidade e da não agudização destas condições. A avaliação clínica e a estratificação de risco buscam compactuar entre profissionais e usuários/as um cuidado baseado na equidade e na integralidade. Estabelecer fluxogramas e frequência de atendimentos para orientação e monitoramento de quadro clínico, bem como garantir acesso à medicação e instigar o automonitoramento e o autocuidado são ferramentas possíveis de serem readequadas em formato presencial e/ou virtual diante do contexto pandêmico (BRASIL, 2020a).

Estratégia 4. O uso de máscara e higienização das mãos com água e sabão ou álcool em gel 70\% é a medida preventiva de maior impacto para evitar a contaminação. Ainda, cobrir nariz e boca com braço ou lenço ao tossir e espirrar, evitar tocar olhos, nariz e boca com as mãos não higienizadas, não compartilhar objetos de uso pessoal, evitar aglomerações e ambientes não ventilados, além de evitar o contato com pessoas contaminadas (distanciamento/isolamento social), são medidas preventivas eficazes para prevenir o contágio da doença e a consequente disseminação (BRASIL, 2020b).

Atuar na perspectiva de educação em saúde é a estratégia fundamental no contexto pandêmico, informando a população e desenvolvendo reflexão crítica diante das formas de autocuidado e não veiculação de Fake News. A educação em saúde consiste em um processo educativo e dialógico de construção de conhecimentos em saúde que tem por finalidade a apropriação temática pela população, contribuindo assim para a autonomia e o protagonismo do cuidado de forma crítica e consciente, fortalecendo saberes coletivos e emancipatórios a partir da realidade local (FALKENBERG, et al., 2014).

Estratégia 5. Na atenção clínica, as modalidades de teleatendimento síncronas e assíncronas surgem para orientar e monitorar a situação de saúde da população, garantindo a continuidade do cuidado e reduzindo agravos de demandas e riscos à saúde. A telerreabilitação é uma modalidade de teleatendimento que objetiva complementar e potencializar as ações de reabilitação através de telecomunicação e tecnologias leves, elaborado através de formação técnico-científica e adequação do tratamento à realidade da pessoa atendida. Observa-se que a autogestão, a adesão e o vínculo terapeutapaciente são impulsionados positivamente nesse formato, contribuindo para maior participação e melhoria de funcionalidade nos casos acompanhados (COUTINHO \& RIBEIRO, 2021). 


\section{CONCLUSÃO}

As intervenções aplicadas nos locais de atuação da equipe multiprofissional possibilitaram a manutenção do cuidado para com a população durante a pandemia, consolidando com isso os princípios e diretrizes do SUS e da política nacional de atenção básica, além de garantir o acesso e o direito à saúde para a população adscrita.

A residência multiprofissional em atenção básica e saúde da família exemplifica a partir das experiências relatadas o potencial que a atenção básica desempenha na rede de saúde, unindo ensino, pesquisa e serviço para o fortalecimento da gestão em saúde e do enfrentamento da pandemia da COVID-19. Espera-se que novos estudos sejam realizados a fim de analisar os impactos a médio e longo prazo que as ações de controle e prevenção no âmbito da atenção básica refletiram no contexto de pandemia.

\section{DECLARAÇÃO DE INTERESSES}

Nós, autores deste artigo, declaramos que não possuímos conflitos de interesses de ordem financeira, comercial, político, acadêmico e pessoal.

\section{REFERÊNCIAS}

AQUINO, E. M. L.; et al. Medidas de distanciamento social no controle da pandemia de COVID-19: potenciais impactos e desafios no Brasil. Ciênc. saúde coletiva, Rio de Janeiro, v. 25, n. 6, p. 24232446, 2020. Disponível em: https://www.scielo.br/j/csc/a/4BHTCFF4bDqq4qT7WtPhvYr/?lang=pt $\&$ format=pdf. Acesso em: 05 jul. 2021.

BRASIL. Ministério da Saúde. Manual - como organizar o cuidado de pessoas com doenças crônicas na aps no contexto da pandemia. Brasília: Ministério da Saúde, 2020a. Disponível em: https://www.gov.br/saude/pt-br/coronavirus/publicacoes-tecnicas/guias-e-planos/manual-de-comoorganizar-o-cuidado-de-pessoas-com-doencas-cronicas-na-aps-no-contexto-da-pandemia.pdf. Acesso em: 05 jul. 2021.

BRASIL. Ministério da Saúde. Protocolo de manejo clínico para o novo coronavírus (2019nCOV). Brasília: Ministério da Saúde, 2020b. Disponível em: https://www.arca.fiocruz.br/bitstream/ icict/40249/2/protocolo_manejo_coronavirus_ms.pdf. Acesso em: 05 jul. 2021.

BRASIL. Ministério da Saúde. Secretaria de Atenção à Saúde. Departamento de Atenção Básica. Política Nacional de Atenção Básica. Brasília: Ministério da Saúde, 2012. Disponível em: https:// bvsms.saude.gov.br/bvs/publicacoes/politica_nacional_atencao_basica.pdf. Acesso em: 05 jul. 2021. CAMPOS, G. W. S.; et al. O papel da atenção primária na rede de atenção à saúde no Brasil: limites e possibilidades no enfrentamento da COVID-19. Cad. Saúde Pública, Rio de Janeiro, v. 36, n. 6, p. 1-7, 2020. Disponível em: http://cadernos.ensp.fiocruz.br/static//arquivo/1678-4464-csp-36- 
06-e00104120.pdf. Acesso em: 05 jul. 2021.

COUTINHO, D. L. L. N.; RIBEIRO, J. D. C. Panorama da telereabilitação na fisioterapia: uma realidade da pandemia COVID-19. In: I Congresso Brasileiro de Medicina e Saúde Online, 2020. Anais... Disponível em: https://doity.com.br/anais/cbmed/trabalho/161524. Acesso em: 05 jul. 2021.

FALKENBERG, M. B.; et al. Educação em saúde e educação na saúde: conceitos e implicações para a saúde coletiva. Ciênc. saúde coletiva, Rio de Janeiro, v. 19, n. 3, p. 847-852, 2014. Disponível em: https://www.scielo.br/j/csc/a/kCNFQy5zkw4k6ZT9C3VntDm/?format=pdf\&lang=pt. Acesso em: 05 jul. 2021.

JUNGES, J. R.; BARBIANI, R.; ZOBOLI, E. L. C. P. Planejamento estratégico como exigência ética para a equipe e a gestão local da atenção básica em saúde. Interface, Botucatu, v. 19, n. 53, p. 265 274, 2015. Disponível em: https://www.scielosp.org/pdf/icse/2015.v19n53/265-274/pt. Acesso em: 05 jul. 2021.

TRIPP, D. Pesquisa-ação: uma introdução metodológica. Educação e Pesquisa, São Paulo, v. 31, n. 3, p. 443-466, set./dez., 2005. Disponível em: https://www.scielo.br/j/ep/a/3DkbXnqBQqyq5bV4TC L9NSH/?format=pdf\&lang=pt. Acesso em: 05 jul. 2021. 


\section{Índice Remissivo}

A

Ações e serviços de saúde 15, 17

Ações multiprofissionais de educação em saúde 6, 22, 24

Alergia a proteína ao leite de vaca (aplv) 88

Anticoncepção pós-coito 95

Anticoncepcional de emergência 95

Aplicativos relacionados à saúde 134, 135, 139

Assistência pré-natal 80

Atenção básica 15, 19, 25, 27, 35, 37, 84, 100, 118, 120, 121, 122

Atenção básica no enfrentamento à covid-19 15

Atenção primária à saúde 15, 22, 23, 35, 36, 41, 49, 120, 124

Atendimento à população 22, 33

Atividades educativas sobre aplv 126

Atopia 88

Autocuidado 18, 44, 62, 64, 66, 73, 76, 123

Autoexame das mamas 39, 42

Avanços tecnológicos 113, 134

$\mathrm{C}$

Câncer de colo uterino 80

Câncer de mama 39, 40, 41, 42, 43, 44, 83, 85

Cárie dentária 71, 72, 73, 74, 75, 77

Cárie dentária na infância 71,73

Cárie na primeira infância (cpi) 71

Ciclo reprodutivo feminino 95, 96, 97, 98, 99

Climatério 40, 80, 82, 84

Comportamento contraceptivo 95

Comportamento sexual dos universitários 95

Contracepção 80,84

Covid-19 7, 12, 14, 15, 16, 17, 19, 20, 31, 32, 36, 45, 46, 47, 48, 49, 50, 52, 53, 54, 57, 60, 61, 92, $115,119,120,122,123,124,125,126,127,128,129$

Crianças 52, 54, 55, 57, 58, 59, 60, 71, 72, 73, 74, 75, 76, 77, 78, 79, 89, 91, 126, 127, 128, 131

Cuidado 28, 31, 62, 65, 93

Cuidados com a saúde $57,73,74,80,84$

Curva epidêmica 15,16 
Diagnóstico precoce à covid-19 15, 17

Direitos reprodutivos 80,83

Doença infecciosa $16,45,46$

Doença multifatorial 71

Doenças pulmonares crônicas 134

Doenças respiratórias crônicas 134

\section{E}

Educação em saúde 20, 22, 44, 53, 55, 71

Educação em saúde bucal 71, 72, 73, 75, 76, 77, 79

Educação em saúde para crianças 52

Educação infantil sobre a aplv 88

Educação interprofissional 22

Ensino e serviço 15

Equipe multiprofissional 9, 15, 62

Estado de calamidade pública 118

F

Falta de acesso às informações 102

Fisioterapia 118, 120, 122

Fisioterapia na atenção básica de saúde 118, 120

G

Gestão em saúde 15, 19

Gravidez indesejada 95, 98, 99, 100

$\mathrm{H}$

Hábitos nocivos 80,81

Hábitos saudáveis $80,81,82$

Hipersensibilidade a leite 127

Hipersensibilidade tipo i 88

Horários de atuação da equipe 15,18

I

Infecções por coronavírus 53

Interface usuário e aplicativos relacionados à saúde 134

Intervenção da telerreabilitação 118 
Leishmania 45, 46, 50

Leishmaniose 45, 46, 47, 49, 50

Leishmaniose cutânea 45

Linha de frente 15, 16, 32, 118

M

Medidas de biossegurança 52, 54, 57

M-health 134, 135, 138, 140

Mídias sociais 127

Mobile health 135, 140, 141

Mudança de hábitos 52, 75

$\mathrm{N}$

Neoplasia 62, 63, 65

$\mathrm{O}$

Obtenção de ists 95, 100

Oncologia 62, 65, 68

Orientação populacional e comunitária 15, 16

$\mathrm{P}$

Parasitas 45, 46

Período pandêmico 52, 56, 57, 58

Prevenção de doenças 53

Prevenção de ist/hiv 80

Prevenção do câncer de mama 39

Processo de saúde-doença 22, 32

Promoção à saúde 6,118

Q

Qualificação dos profissionais de saúde 22, 23

$\mathrm{R}$

Reação de hipersensibilidade 88

Reações alérgicas 126, 127

Recomendações sanitárias 15, 118

Rede hospitalar 15, 16

Redes de comunicação digital 102

Redes de internet 102, 104

Rede social instagram ${ }^{\circledR} 126$ 
Residência multiprofissional em saúde 15, 22, 23, 24, 35, 36

Residência multiprofissional em saúde da família 15

$\mathrm{S}$

Saúde bucal 71, 72, 73, 74, 75, 76, 78, 79

Saúde da família 6, 15, 22, 24, 25, 35, 36, 42, 118, 121, 122

Saúde da mulher 39, 40, 44, 80, 81

Saúde indígena 39

Saúde mental 28, 30, 31, 37, 53, 56, 57, 59, 60, 102, 103, 104, 106, 107, 108, 110, 111, 114, 115

Saúde pública 39, 40, 41, 125, 128, 135

Serviço social 22

Sistemas de saúde no brasil e no mundo 15, 16

Sistema único de saúde (sus) 15, 16, 17, 22, 23, 34, 35, 42, 91, 108

Smartphone applications 135

$\mathrm{T}$

Tecnologias móveis 134, 136

Tecnológicas de saúde 102

Teleconsulta 15, 121, 124

Telerreabilitação 15,118

Terapia medicamentosa 62,66

Terapia ocupacional 118,120

Tratamento intralesional de lcl 45, 47, 49

Tratamento oncológico 62, 63, 64, 65

$\mathrm{U}$

Unidades de saúde da família 22

Uso de máscara e álcool em gel 15

Utilização de aplicativos relacionados a promoção da saúde 134

V

Viabilidade do uso de m-health apps 134

Vínculo médico-paciente 45, 48

Violência contra a mulher 80,82 


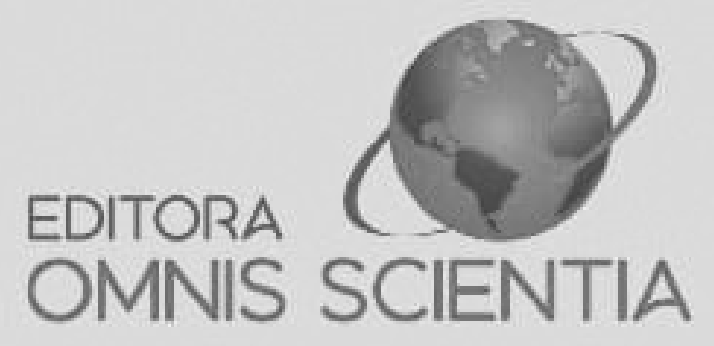

editoraomnisscientia@gmail.com M https://editoraomnisscientia.com.br/ @editora_omnis_scientia (0) https://www.facebook.com/omnis.scientia.9 $\subsetneq$ +55 (87) 9656-3565 (C) 


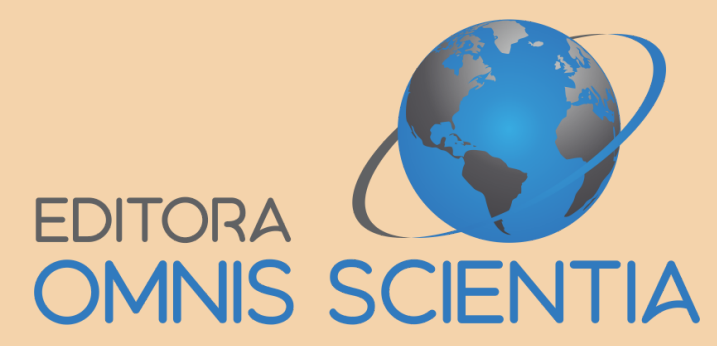

editoraomnisscientia@gmail.com M https://editoraomnisscientia.com.br/

@editora_omnis_scientia (0)

https://www.facebook.com/omnis.scientia.9 $\bigoplus$ +55 (87) 9656-3565 